\title{
La différence subtile entre «compliqué» et «complexe»
}

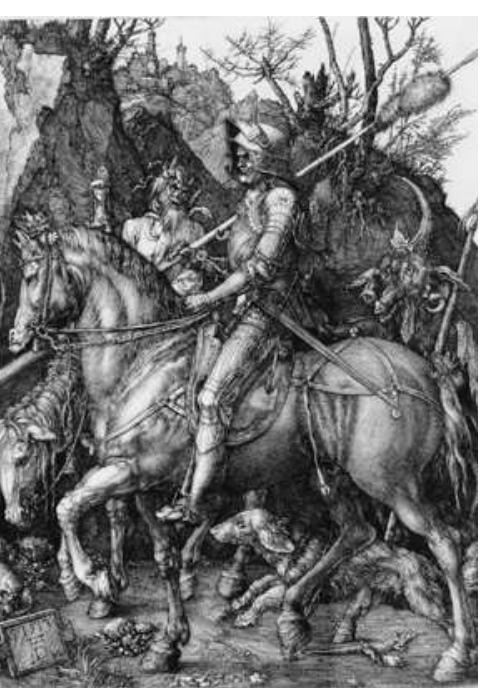

La perte de complexité d'un système peut mener soit au chaos, soit à la rigidité. Albrecht Dürer, 1513: Ritter, Tod und Teufel (Albertina, Wien).
En septembre 2001, plusieurs articles dans le British Medical Journal avaient attiré l'attention sur le phénomène de la complexité en médecine et en santé. Par la suite, le sujet des systèmes complexes en médecine générale a été magistralement traité dans le livre «Complexity in Primary Care» de Kieran Sweeny [1] et la complexité a été choisie comme thème principal de la conférence de la Wonca à Bâle en 2009 et... trois ans auparavant comme sujet de la leçon d'adieu du soussigné [2].

Un système complexe se caractérise par ses capacités d'autorégulation et d'adaptation à d'autres systèmes. On ne peut le décomposer dans ses parties et le restructurer sans une perte irrémédiable de sa structure. Un système complexe est sensible aux conditions antérieures, donc au passé: il change dans le temps. Des interventions minimes peuvent provoquer des réponses inattendues et importantes. Enfin, la perte de complexité d'un système peut soit mener au chaos, soit à la rigidité.

La reconnaissance que les systèmes physiologiques et, par conséquent, nos patients peuvent être considérés comme des systèmes complexes, a des répercussions importantes: tout d'abord, l'approche linéaire, voir causale, des phénomènes de la maladie importante pour comprendre la physiologie - ne suffit pas nécessairement pour la guérir. La maladie doit être comprise comme une perte de complexité et non pas, ainsi que nous l'imaginons, comme un accroissement de complexité. Le rythme sinusal, expression parfaite de complexité, peut en cas de maladie évoluer vers une fibrillation (chaos), voire un arrêt (rigidité et mort). C'est donc le sujet (ou le cœur) sain qui est dans un état de complexité maximale, voire optimale. Quand nous intervenons chez un patient, nous devons nous rendre compte qu'un système complexe est sensible aux conditions antérieures, qu'il a en quelque sorte une mémoire: d'une part la restitutio ad integrum dans le sens strict n'est pas possible et d'autre part chaque patient réagira différemment selon ses expériences antérieures, car l'organisme complexe aura adapté sa structure entre-temps. Enfin, nous devons réaliser que la rencontre entre patient et médecin peut être comprise comme celle de deux systèmes complexes, chacun avec son passé: elle sera chaque fois renouvelée et changera dans le temps, et qui n'en a pas fait l'expérience? - nos interventions, aussi minimes soient-elles, peuvent avoir des conséquences inattendues.

Malgré une littérature abondante sur les systèmes complexes, tous les auteurs n'ont pas compris vérita- blement la signification de la complexité. Il existe une confusion (comme dans le langage commun) entre les termes «complexe» et "compliqué». Ainsi, plusieurs auteurs ont essayé de définir la complexité des patients selon le nombre de leurs comorbidités [3, 4]. D'autres ont essayé d'y ajouter des facteurs socio-économiques pour distinguer le patient complexe du patient non complexe [5]. Cette approche est typique de la pensée linéaire en médecine. On dissèque et on oublie que le réassemblage des parties ne constitue jamais l'ensemble. Paradoxalement, les patients âgés considérés comme complexes, car atteints de multiples maladies, ont en fait perdu de leur complexité, ils ne peuvent plus s'adapter aux influences intérieures ou extérieures et ils sont aussi beaucoup plus sensibles à nos interventions: un diurétique qui induira, chez une personne jeune, au plus une diurèse accrue, peut déclencher, chez le patient âgé et souffrant de comorbidités, hyponatrémie, œè̀me cérébral, hypotension, chute, fracture, embolie pulmonaire et décès... Ces patients ne sont pas plus complexes, mais ils sont devenus plus compliqués!

Rappelons que complication vient du latin «complicatio», qui veut dire enroulement, multiplication, alors que «complexus» signifie enveloppement, embrassement. Une affaire compliquée se composera donc de nombreux éléments, qui peuvent être détaillés par une analyse linéaire, souvent utilisé par le spécialiste, alors qu'un système complexe réunira plusieurs éléments différents et demandera une approche holistique, domaine du généraliste. Essayons donc de faire la subtile différence [6]!

Hans Stalder*

\section{Références}

1 Sweeny K. Complexity in primary care. Oxford: Radcliffe Publishing; 2006.

2 Stalder H. Médecine générale - pensée linéaire et complexité. Rev Med Suisse 2006;2:2150-3.

3 De Jonge P, Huyse FJ, Stiefel FC. Case and care complexity in the medically ill. Med Clin North Am. 2006;90:679-92.

4 Weiss KB. Managing complexity in chronic care: an overview of the VA state-of-the-art (SOTA) conference. J Gen Intern Med. 2007;22 Suppl 3:374-8.

5 Grant RW, Ashburner JM, Hong CS, Chang Y, Barry MJ, Atlas SJ. Defining patient complexity from the primary care physician's perspective: a cohort study. Ann Intern Med. 2011;155:797-804.

6 Waldvogel FA, Perone N, Schussele-Filliettaz S, et al.: Les malades complexes: de la théorie des systèmes complexes à une prise en charge holistique et intégrée. Rev Med Suisse. 2012;8:1022-4 